\title{
AVALIAÇÃO DO GPR NA ESTIMATIVA DA BATIMETRIA DE UMA CAIXA DE DECANTAÇÃO EMPREGANDO O APLICATIVO GRATUITO “GPR BATHYMETRY" NA ANÁLISE DOS RADARGRAMAS
}

\section{Evaluation of the GPR in the bathymetry estimate of a decanting water treatment plant using the free software "GPR Bathymetry" in the radargrams analysis}

\author{
Sandro Henrique de Faria ${ }^{1,2}$ \\ Dalto Domingos Rodrigues ${ }^{2}$ \\ Nilcilene das Graças Medeiros ${ }^{2}$ \\ Paulo Roberto Antunes Aranha ${ }^{3}$
}

\begin{abstract}
${ }^{1}$ Instituto Federal de Educação, Ciência e Tecnologia do Sudeste de Minas, Juiz de Fora - MG, Brasil, Email: sandro.faria@ifsudestemg.edu.br.
\end{abstract}

2Universidade Federal de Viçosa - Viçosa - MG, Brasil, Emails: dalto@ufv.br, nilcilene.medeiros@ufv.br.

3Universidade Federal de Minas Gerais, Belo Horizonte - MG, Brasil, Email: aranha@igc.ufmg.br.

\section{Resumo:}

Este trabalho tem como objetivos avaliar o desempenho do GPR (Ground Penetrating RADAR) para aplicações batimétricas em ambiente controlado e do aplicativo "GPR Bathymetry", desenvolvido para análise de radargramas batimétricos. $\mathrm{O}$ estudo foi realizado em uma caixa de decantação da estação de tratamento de água da qual se tem o "as-built", obtido com estação total, que será empregado nos testes de validação. Realizaram-se três testes de comparação: comparações pontuais; entre Modelos Digitais de Elevação (MDE) gerados a partir do interpolador Topo to Raster; e entre os volumes calculados a partir dos MDE's. Na comparação pontual a maior amplitude de discrepância entre as médias foi de $8 \mathrm{~cm}$. Para a amostra de discrepâncias entre os MDE's, a amplitude foi de $9 \mathrm{~cm}$, média de $2 \mathrm{~cm}$, acurácia estimada de $4 \mathrm{~cm}$ e o RMS de $3 \mathrm{~cm}$. Na comparação volumétrica obteve-se o valor de $800,6 \mathrm{~m}^{3}$ com dados de referência e $806,4 \mathrm{~m}^{3}$ com dados do GPR, resultando numa diferença de $1 \%$ entre os modelos. Conclui-se que para um reservatório construído em concreto armado, profundidades variando de 3 a 3,5 metros, o levantamento do relevo submerso empregando um GPR apresentou resultados promissores e um MDE com acurácia estimada de $4 \mathrm{~cm}$ e discrepâncias de até $9 \mathrm{~cm}$.

Palavras chave: GPR, MDE, Batimetria, Experimento, Software gratuito.

\begin{abstract}
:
This paper aims to evaluate the performance of the GPR (Ground Penetrating Radar) for bathymetric applications in a controlled environment, as well as evaluate the "GPR Bathymetry" software designed to bathymetric radargrams analysis. The study was realized in a decanting water treatment plant (WTP), where has the as-built obtained with total station, it will be used in validation tests. Was realized three comparison tests: from points comparisons; from Digital Elevation Models (DEM)
\end{abstract}


generated from interpolating Top to Raster; and between volumes calculated from DEMs. In the point comparison, the greatest difference between averages was $8 \mathrm{~cm}$. For the discrepancies sample between DEMs, the amplitude was $9 \mathrm{~cm}$, average of $2 \mathrm{~cm}$, estimate accuracy of $4 \mathrm{~cm}$ and RMS equal to $3 \mathrm{~cm}$. In the volumetric comparison, we obtained the amount of $800.6 \mathrm{~m}^{3}$ with reference data and $806.4 \mathrm{~m}^{3}$ with the GPR data, resulting in a $1 \%$ difference between models. We conclude that for a reservoir built in reinforced concrete, depths ranging from 3 to 3.5 meters, the survey of the bottom relief employing a GPR showed promising results and a DEM with an estimate accuracy of $4 \mathrm{~cm}$ and discrepancies that may reach $9 \mathrm{~cm}$.

Keywords: GPR, DEM, bathymetry, experiment, free software.

\section{Introdução}

O monitoramento das represas de abastecimento de água, não só no quesito de qualidade da água, mas também no sentido quantitativo, isto é, volumétrico, é uma preocupação para as autoridades públicas pois o assoreamento afeta a capacidade de armazenamento do reservatório. Dentro desta ótica surge a necessidade da utilização de novas técnicas e o aperfeiçoamento de outras para o monitoramento da profundidade da lâmina d’água.

A técnica que trata da determinação e representação gráfica do relevo de áreas submersas é chamada de Batimetria. Com o avanço das tecnologias os levantamentos batimétricos estão sendo, atualmente, realizados com o auxílio de receptores GNSS (Global Navigation Satellite System) para a determinação do posicionamento planimétrico e ecobatímetros para medição da profundidade.

Atualmente, os ecobatímetros monofeixe e multifeixe são os mais empregados para determinação da profundidade da lâmina d'água. Estes equipamentos consistem basicamente de uma fonte emissora de sinais acústicos e um dispositivo interno que mede o intervalo de tempo entre o instante da emissão do sinal e o instante de recepção do eco pelo sensor. Parte do som emitido é captado pelo dispositivo receptor após viajar pelo meio liquido sofrendo reflexão, transmissão e espalhamento. Esse sinal é então convertido em sinais elétricos e o intervalo de tempo medido (Blondel e Murton, 1997). A sondagem é calculada multiplicando este intervalo de tempo pela velocidade do som na água e dividindo-se por dois, já que se trata de tempo duplo de reflexão, fornecendo desta maneira informações de profundidade no local.

O GPR é uma ferramenta não invasiva, contínua e de alta velocidade na coleta de dados que tem sido usada para mapear as condições da subsuperfície em uma vasta variedade de aplicações (Botelho, 1986; Aranha, et al, 2002; Annan, 2003; Castelo Branco e Castro, 2003; Shiraiwa, et al, 2004; Saarenketo, 2006; Gandolfo, 2007; Faria, 2010).

Os sistemas de GPR transmitem pulsos eletromagnéticos em um meio e quando estes encontram um contraste significativo entre as propriedades elétricas dos materiais acima e abaixo desta interface, parte da energia é refletida de volta enquanto o restante seguirá adiante sendo refletida novamente por outra interface ou absorvida totalmente. A imagem obtida é denominada radargrama e constitui a base para se interpretar e construir o perfil da subsuperfície. A qualidade do radargrama pode variar em virtude das características dos pulsos refletidos, refratados e difratados, que são condicionados às propriedades dielétricas do material investigado e da interação deste com o meio onde está inserido, como também da frequência da antena utilizada. Estes sistemas de GPR possuem tipicamente os seguintes componentes: um gerador de pulsos, sendo este pulso com frequência e potência específica; uma ou mais antenas, que transmitem o pulso para a subsuperfície e um classificador e registrador de 
dados, que capturam e registram os sinais refletidos pelo meio. A Figura 01 apresenta modo de obtenção de dados com antenas de contato também conhecidas como ground coupled antennas.

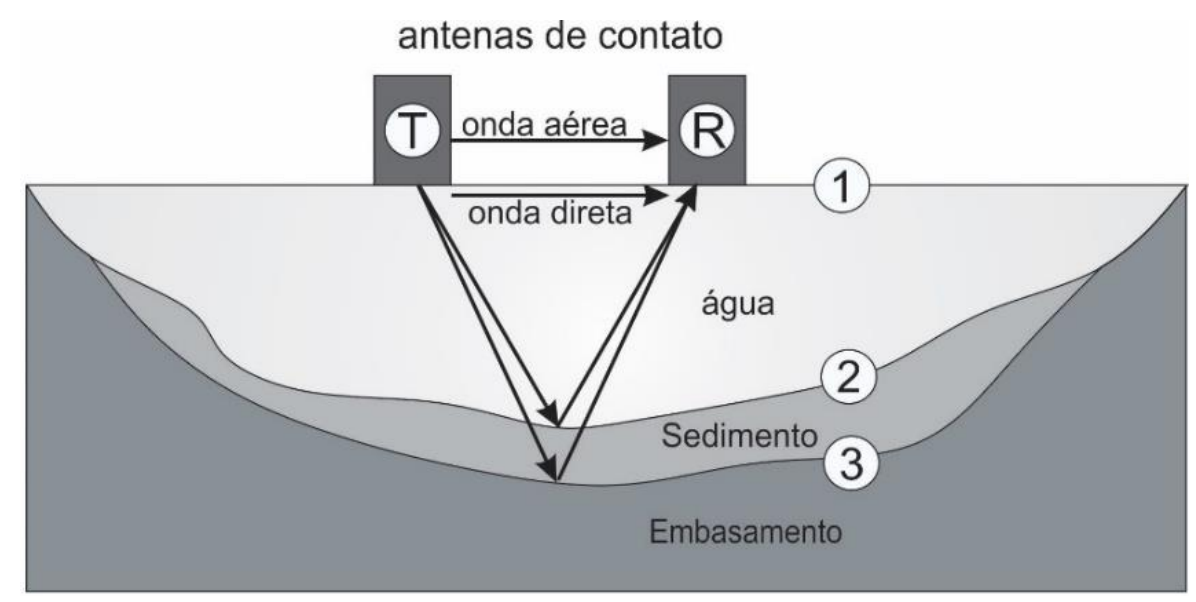

Figura 1: Princípio básico da técnica GPR onde T é a antena transmissora e $\mathrm{R}$ a antena receptora e 1, 2 e 3 as interfaces de reflexão.

Para o modelo da Figura 1, a interface 1 representa a interface do ar com a lâmina d’água, 2 representa a interface da lâmina d'água com sedimento e 3 representa a interface do sedimento com o embasamento. As reflexões nas interfaces 1, 2 e 3 do modelo da Figura 1 podem ser visualizados na Figura 2.

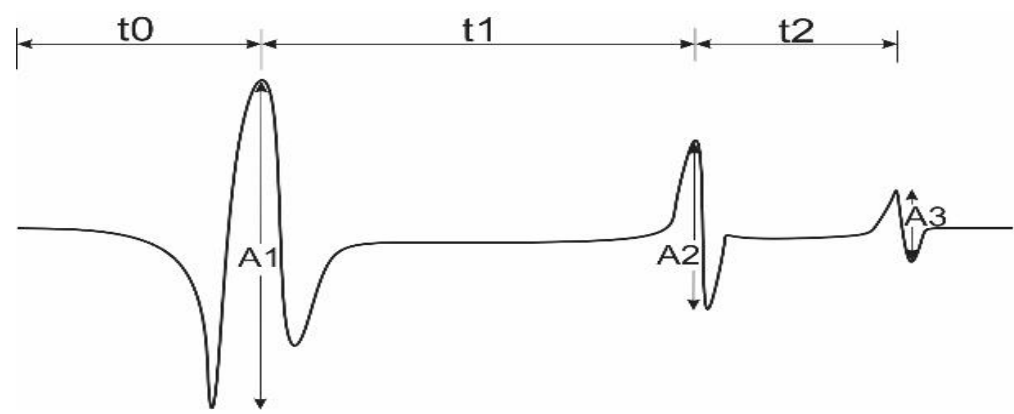

Figura 2: Exemplo do traço de georadar.

Pela Figura 2, A1 é a amplitude total da onda que viaja na parte superior da lâmina d'água (onda direta), A2 a amplitude total de reflexão na interface entre a lâmina d'água e o sedimento e A3 a amplitude de reflexão entre o sedimento e o embasamento. to é o intervalo de tempo gasto pela onda aérea e onda direta, t1 é o intervalo de tempo de propagação do sinal na camada de água e t2 é o intervalo de tempo gasto para percorrer a camada ocupada pelo sedimento, lembrando que estes intervalos de tempos são referentes à ida e volta do sinal.

Os dados registrados pela reflexão da onda nas interfaces mostradas no modelo da Figura 1 podem ser interpretados para se deduzir as características do substrato. $O$ traço do georadar é o pulso captado pela antena receptora após o pulso emitido ser refletido e refratado nas interfaces da subsuperfície e percorrer o caminho ascendente indo direto para a antena receptora (Figura 2). O radargrama é uma matriz de dados onde cada coluna corresponde a um traço obtido na posição investigada e as linhas correspondem ao tempo de ida e volta do sinal emitido.

O princípio básico de funcionamento do GPR é que conhecendo o intervalo de tempot e a velocidade médiav de propagação do pulso ao longo do caminho percorrido, é possível determinar a distância $d$ (Equação 1) em que um determinado objeto refletor está da fonte emissora do pulso (Saarenketo, 2006). A velocidade de propagação da onda eletromagnética emitida pelo GPR no ar pode ser tomada como sendo igual à velocidade de propagação da luz no vácuo $(0,3 \mathrm{~m} / \mathrm{ns})$. A velocidade da onda 
eletromagnética na água destilada é de $0,033 \mathrm{~m} / \mathrm{ns}$, enquanto na água do mar a velocidade cai para 0,01 $\mathrm{m} / \mathrm{ns}$ (Annan, 2003).

$$
d=\frac{v \times t}{2}
$$

\section{Metodologia}

Para realização deste trabalho foi desenvolvido um aplicativo gratuito, em Matlab ${ }^{\circledR}$, na versão 2012b, chamado "GPR Bathymetry", para a análise dos radargramas. O objetivo do desenvolvimento desse aplicativo é de disponibilizar ao usuário que trabalha com o GPR uma ferramenta gratuita e inicialmente desenvolvida para aplicações batimétricas. Ainda na primeira versão, conta com ferramentas de visualização, interpoladores de superfície, filtros de detecção de bordas, filtros de suavização, filtros morfológicos, set time zero, remove background, edição de dados, ganhos, cálculos de áreas e volumes, espacialização de radargramas em X, Y e Z e classificação manual. Está em desenvolvimento módulos para classificação semiautomatizada, filtros no domínio da frequência, e multivisualização de radargramas georreferenciados.

\subsection{Caracterização da área de estudo}

Para a realização do experimento os dados foram coletados em uma caixa de decantação da Estação de Tratamento de Água (ETA), situada na Universidade Federal de Viçosa (UFV). A caixa de decantação é construída em concreto armado e possui aproximadamente 18 metros de diâmetro. A parte mais rasa possui em média 2,989 metros e a mais profunda 3,548 metros. O local foi escolhido por apresentar características favoráveis a uma área experimental controlada e por já se ter dados de levantamento topográfico realizados com estação total, levantamentos estes extraído de Carmo (2014).

A Figura 3 apresenta o aspecto da estação de tratamento de água e do levantamento realizado com o GPR (Sistema RAMAC desenvolvido pela MALA Geoscience), com uma antena unshielded $200 \mathrm{MHz}$. Utilizou-se um fio de nylon para puxar o flutuador mantendo-o alinhado.

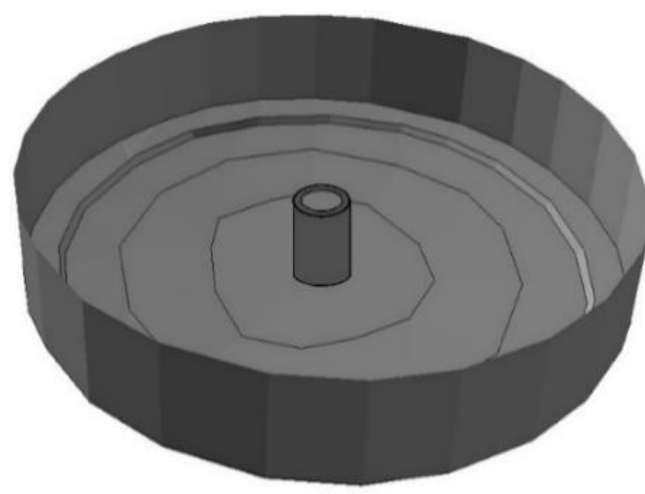

(a)

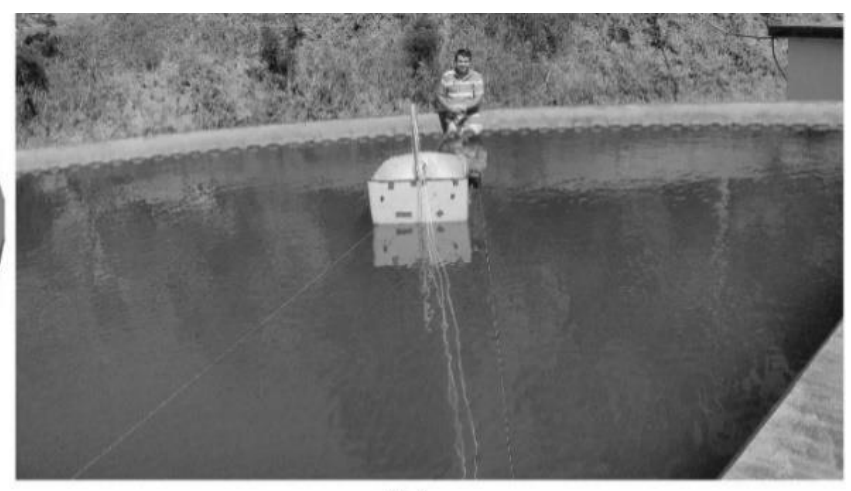

(b)

Figura 3: (a) Modelo 3D da Caixa de decantação modificado de Carmo (2014). (b) Foto mostrando o levantamento de uma seção. 


\subsection{Coleta dos dados}

Para a realização deste ensaio foram determinadas previamente 13 seções distribuídas radialmente de maneira uniforme. A Figura 4 mostra a distribuição dos pontos amostrados na ETA com estação total, os quais serão adotados como referência, bem como as direções dos 13 perfis levantados com o GPR.

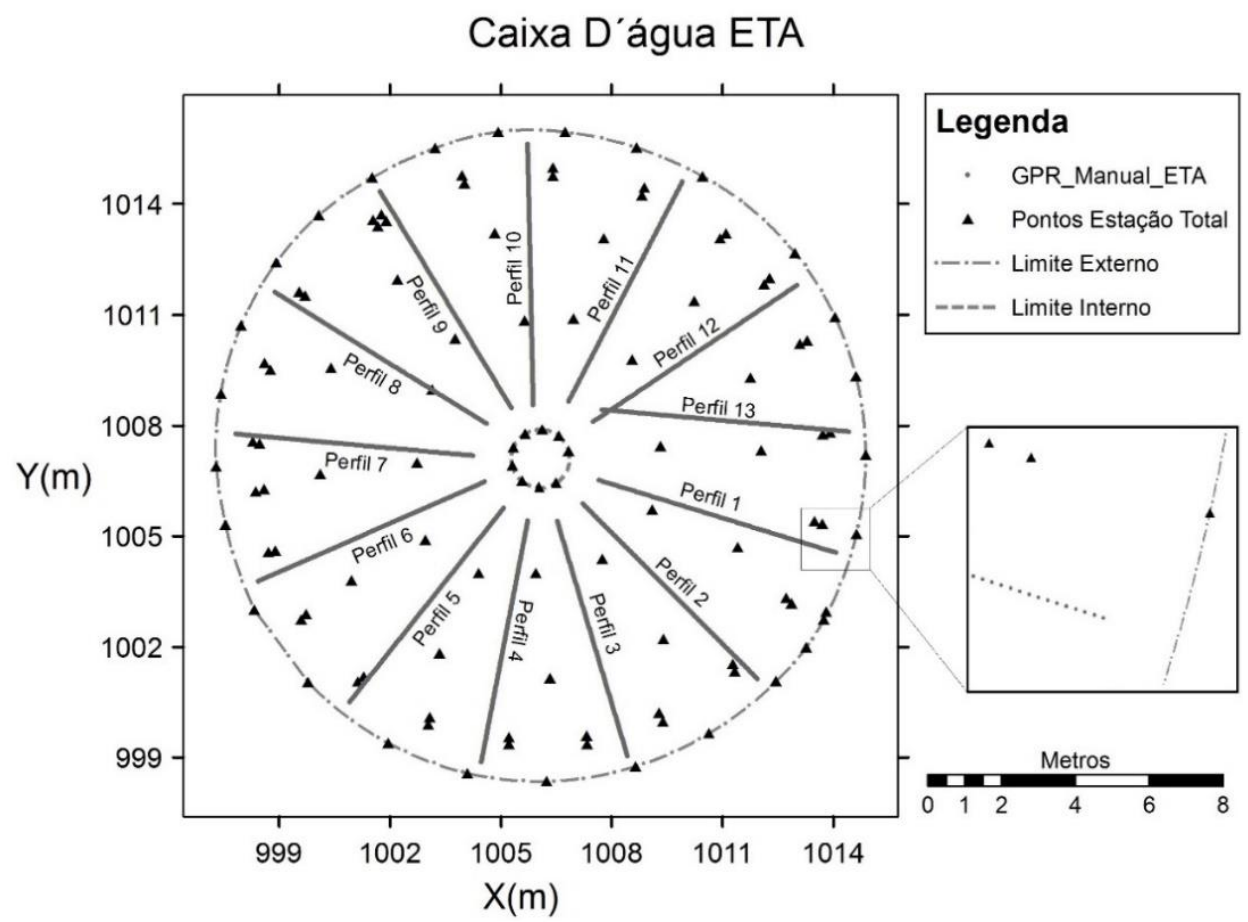

Figura 4: Pontos amostrados pela estação total e as direções dos 13 perfis levantados com o GPR.

\subsection{Análise dos radargramas}

Os perfis foram analisados no aplicativo "GPR Bathymetry", aplicando ao radargrama a função "set time zero" para a localização e remoção da onda direta, que está na superfície da água, e a função "remove background" para remoção dos ruídos. A Figura 5 apresenta o perfil 1 no estado bruto e a Figura 6 apresenta o aspecto do mesmo perfil após o processamento. 


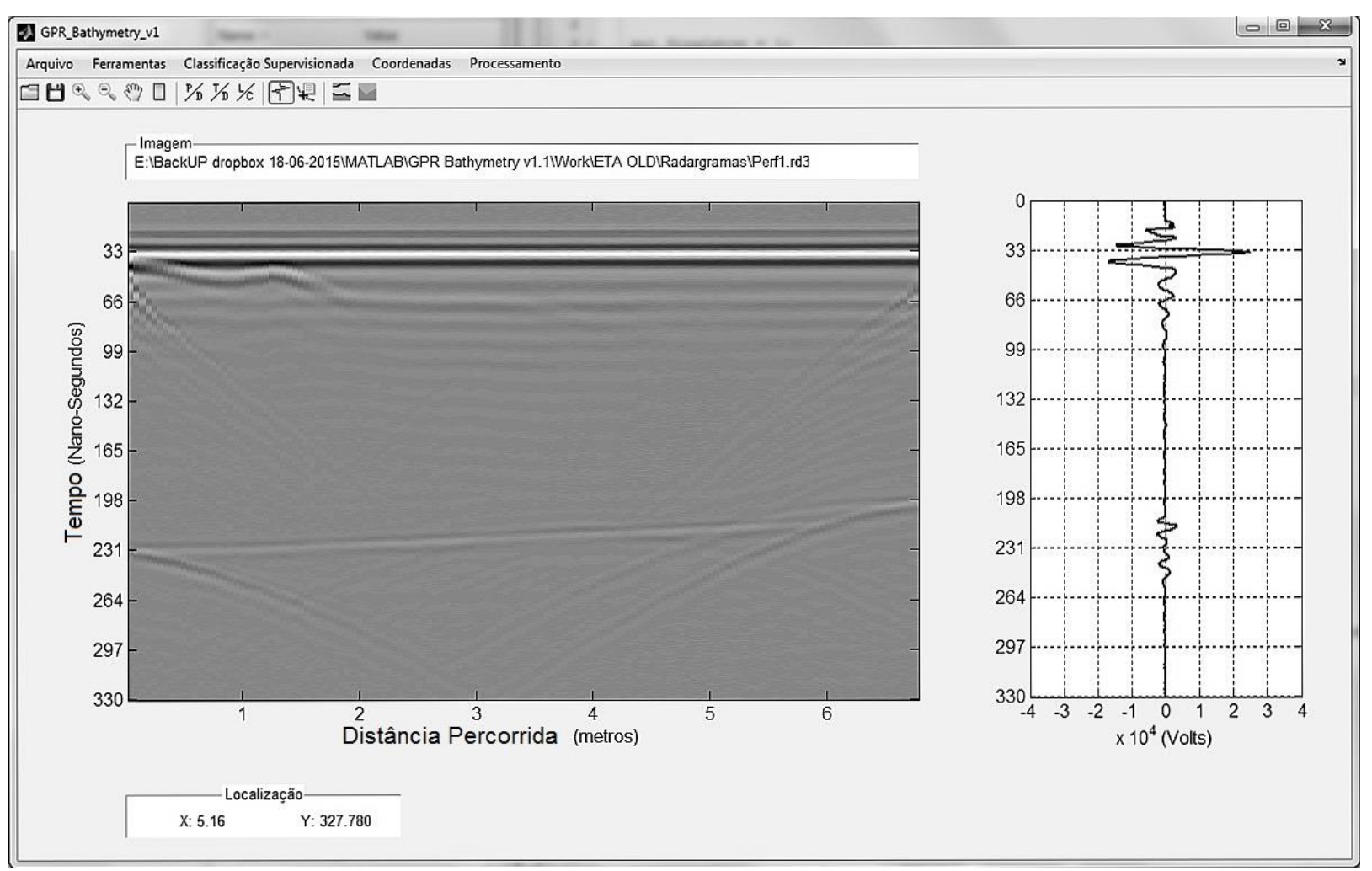

Figura 5: Radargrama do perfil 1 exibido na janela principal do "GPR Bathymetry".

\subsection{Identificação manual do fundo da caixa e do ponto de controle.}

Para a identificação manual da reflexão do pulso captado, foi utilizada uma ferramenta de edição disponível no "GPR Bathymetry". Esta ferramenta possui funcionalidades de inserir linhas, polilinhas, splines e polígonos, tornando possível uma classificação manual, como mostrado na Figura 6.

Da Figura 6 é possível extrair, para uma determinada posição, o intervalo de tempo de propagação do sinal (ida e volta). Conhecendo-se a profundidade nesta mesma posição, medida por outro método qualquer, é possível determinar a velocidade de propagação do sinal no meio, supondo-a constante, empregando a Equação 1. Na Figura 6 é destacada uma posição a 2 metros do centro da caixa, sobre o perfil, em que o intervalo de tempo foi de $209 \mathrm{~ns}$ e a profundidade observada de 3,386 m. Com estas informações é possível determinar o valor da velocidade (Equação 2).

$$
v=\frac{2 d}{t}=\frac{2 \times 3,39 \mathrm{~m}}{209 \mathrm{~ns}}=0,0324 \mathrm{~m} / \mathrm{ns}
$$

O mesmo procedimento foi adotado para os perfis 5 e 10 , obtendo como velocidades 0,0320 e 0,0328 $\mathrm{m} / \mathrm{ns}$. O valor médio obtido de $0,0324 \mathrm{~m} / \mathrm{ns}$ foi muito próximo do valor de $0,033 \mathrm{~m} / \mathrm{ns}$ encontrado nas bibliografias para a água destilada (Annan, 2003). 


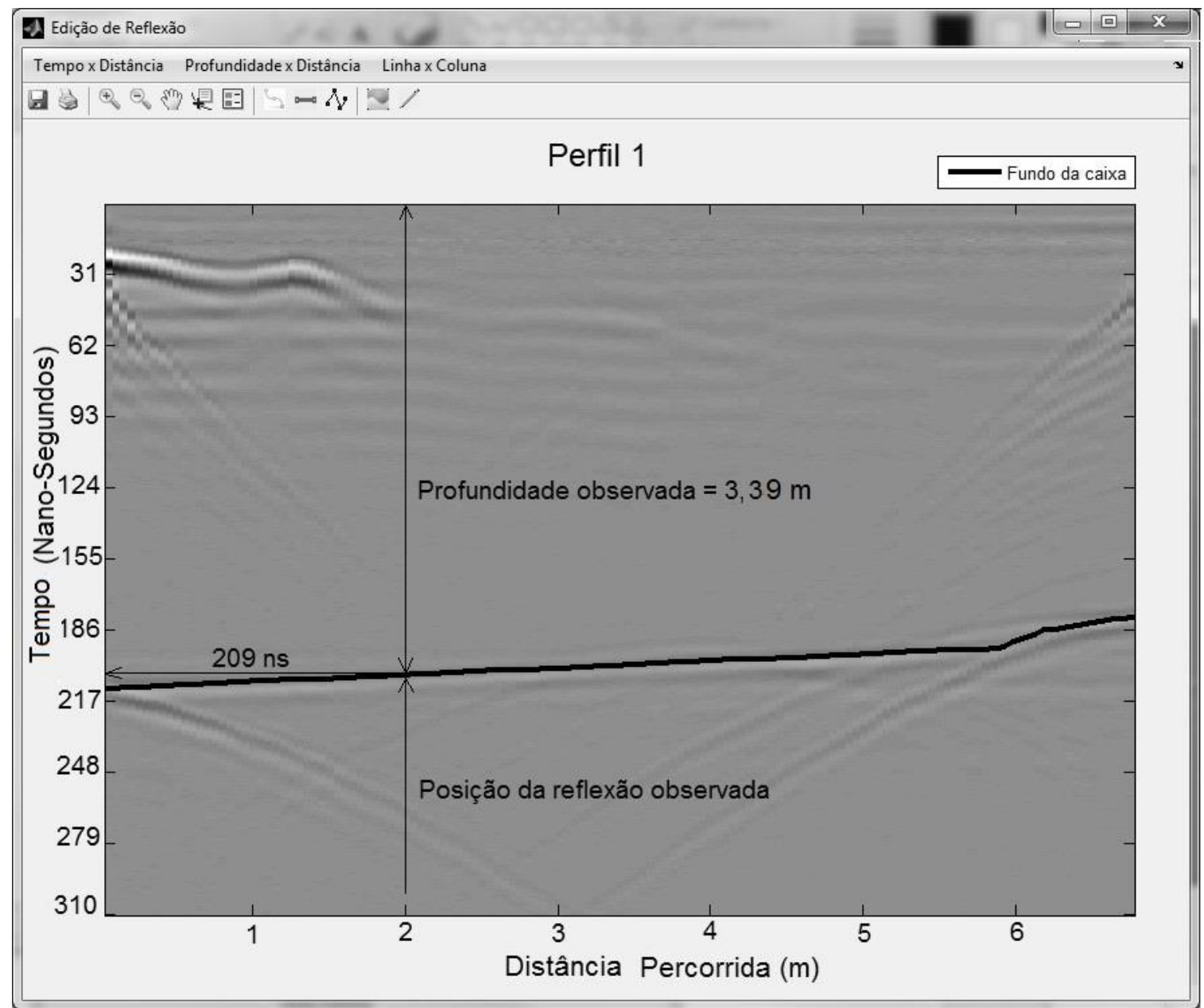

Figura 6: Profundidade e tempo de propagação em uma mesma posição.

Uma vez inserido o valor da velocidade média de propagação do sinal, na água da caixa em estudo, no aplicativo, torna-se possível a conversão do radargrama de tempo (ns) para profundidade (metros). Também é possível exportar um arquivo, na extensão *.zzz, contendo os valores de profundidade para cada traço a partir da linha inserida pelo usuário na demarcação da reflexão do sinal com o piso de concreto da ETA (Figura 6). Este arquivo será usado na etapa de espacialização do radargrama.

\subsection{Espacialização dos radargramas}

Durante o levantamento dos dados foram medidas, em um sistema de referência topográfico, as coordenadas $\mathrm{X}, \mathrm{Y}$ e $\mathrm{Z}$ dos pontos inicial e final de cada radargrama utilizando estação total, uma vez que os perfis seguiram um determinado alinhamento (Figura 4). De posse dessa informação torna-se possível a atribuição das coordenadas planas $\mathrm{X}, \mathrm{Y}$ e da coordenada $\mathrm{Z}$ para cada traço do radargrama, correspondente à superfície. Para isso foi desenvolvida uma ferramenta que calcula o comprimento de cada radargrama, o seu número de traços e realiza uma interpolação, que pode ser linear ou cúbica (caso se tenha mais de 2 pontos de controle), de modo que os traços estejam igualmente espaçados entre si. Os pontos de controle podem ser inseridos manualmente em uma tabela de entrada de dados ou por meio de um arquivo no formato de texto com extensão *.xyz. O aspecto dos pontos de controle e dos pontos interpolados podem ser visualizados na Figura 7. As coordenadas interpoladas da superfície também podem ser salvas no mesmo formato ou acrescentadas no arquivo *.RAD. 


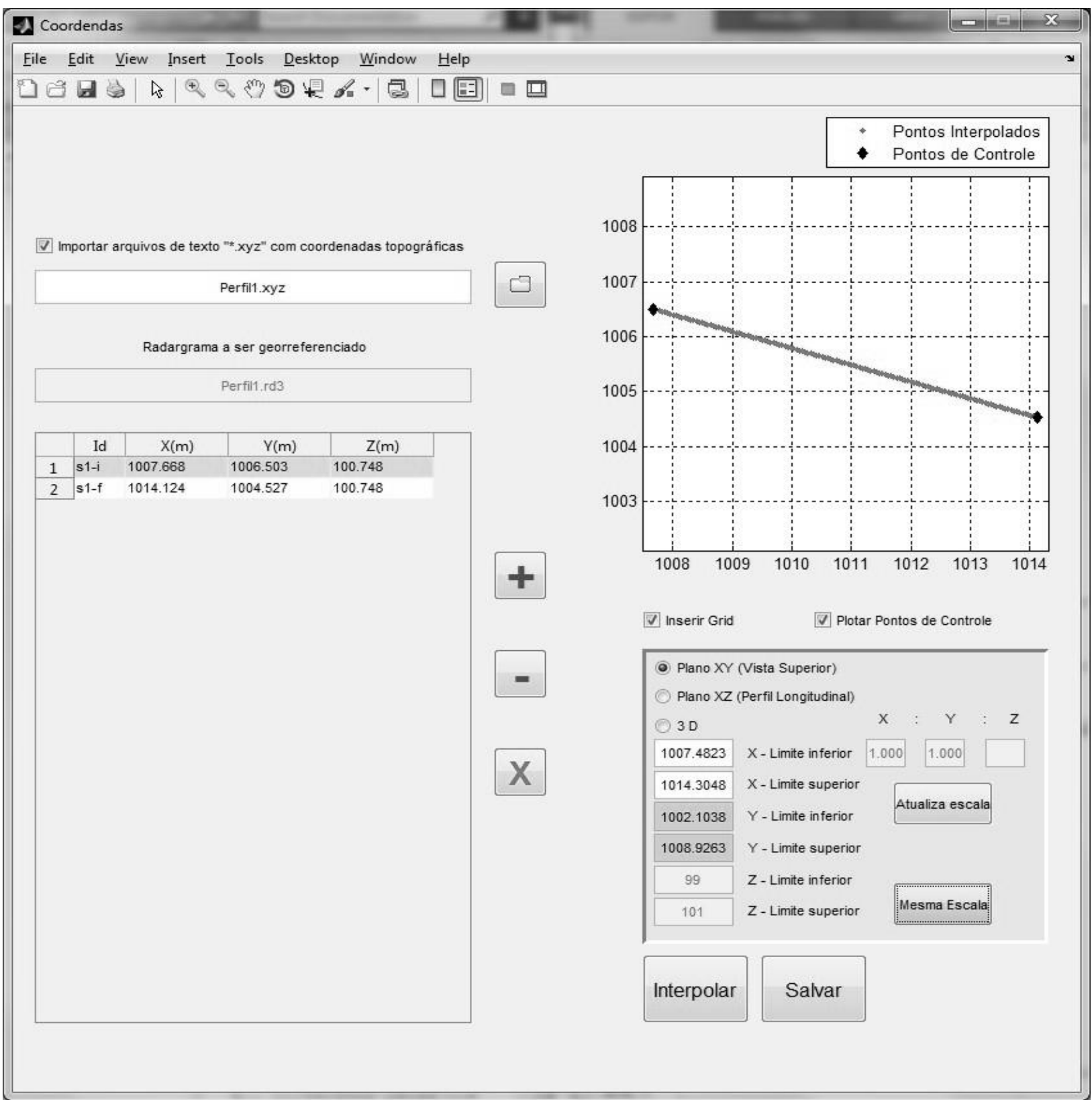

Figura 7: Espacialização de todos os traços do radargrama para o perfil 1.

De posse das coordenadas topográficas (X, Y, Z), na superfície, de cada traço (arquivos *.xyz) e dos valores de profundidade para cada ponto onde ocorreu a reflexão (arquivos *.zzz), é possível determinar as coordenadas topográficas $(\mathrm{X}, \mathrm{Y}, \mathrm{Z})$ de cada ponto da reflexão no interior da caixa d'água. Para realizar essa tarefa, foi desenvolvida uma ferramenta cujos resultados são apresentados na Figura 8, onde se vê tabelas com os dados de entrada, os arquivos *.xyz e *.zzz, e os dados de saída, que são as coordenadas tridimensionais de todos os traços refletidos no fundo da ETA, para as 13 radiais. Os dados de saída podem ser gravados no formato de texto *.xyz do "GPR Bathymetry", *.txt do ArcGis e *.xlsx do excel. 


\begin{tabular}{|c|c|c|c|}
\hline & \multicolumn{2}{|c|}{ Buscar Arquivo(s) " *.xyz" } & \\
\hline & Nome & Traços & \\
\hline 1 & Perfil1.xyz & 135 & A \\
\hline 2 & Perfil2.xyz & 135 & 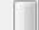 \\
\hline 3 & Perfill.$x y z$ & 134 & \\
\hline 4 & Perfil4.xyz & 135 & \\
\hline 5 & Perfil5.xyz & 135 & 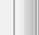 \\
\hline 6 & Perfil6.xyz & 136 & 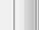 \\
\hline 7 & Perfil7.xyz & 130 & $E$ \\
\hline 8 & Perfil8.xyz & 136 & 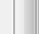 \\
\hline 9 & Perfil9.xyz & 138 & 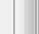 \\
\hline 10 & Perfil10.xyz & 142 & 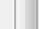 \\
\hline 11 & Perfil11.xyz & 135 & 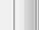 \\
\hline 12 & Perfil12.xyz & 134 & L \\
\hline \multirow[t]{3}{*}{13} & Perfil13.xyz & 135 & + \\
\hline & \multicolumn{3}{|c|}{ Buscar Arquivo(s) " *.zzz" } \\
\hline & Nome & Traços & \\
\hline 1 & Perf1_TimeZero_RB-30a80.zzz & 135 & . \\
\hline 2 & Perf2_TimeZero_RB-30a80.zzz & 135 & r \\
\hline 3 & Perf3_TimeZero_RB-30a80.zzz & 134 & \\
\hline 4 & Perf4_TimeZero_RB-30a80.zzz & 135 & \\
\hline 5 & Perf5_TimeZero_RB-30a80.zzz & 135 & \\
\hline 6 & Perf6_TimeZero_RB-030a80.zzz & 136 & \\
\hline 7 & Perf7_TimeZero_RB-30a80.zzz & 130 & $\equiv$ \\
\hline 8 & Perf8_TimeZero_RB-30a80.zzz & 136 & \\
\hline 9 & Perf9_TimeZero_RB-30a80.zzz & 138 & \\
\hline 10 & Perf10_TimeZero_RB-30a80.zzz & 142 & \\
\hline 11 & Perf11_TimeZero_RB-30a80.zzz & 135 & \\
\hline 12 & Perf12_TimeZero_RB-30a80.zzz & 134 & L \\
\hline 13 & Perf13_TimeZero_RB-30a80.zzz & 135 & - \\
\hline
\end{tabular}

\begin{tabular}{|c|c|c|c|c|c|}
\hline & \multicolumn{4}{|c|}{ Gravar Dados } & \\
\hline & ID & $X(m)$ & $Y(m)$ & $Z(m)$ & \\
\hline 120 & \multicolumn{2}{|c|}{ Perf1_1... 1013.359} & 1004.761 & 97.577 & A \\
\hline 121 & \multicolumn{2}{|c|}{ Perf1_1... 1013.407} & 1004.747 & 97.603 & \\
\hline 122 & \multicolumn{2}{|c|}{ Perf1_1 . 1013.454} & 1004.732 & 97.620 & \\
\hline 123 & \multicolumn{2}{|c|}{ Perf1_1 $\ldots$ 1013.502 } & 1004.717 & 97.646 & \\
\hline 124 & \multicolumn{2}{|c|}{ Perf1_1... 1013.550} & 1004.703 & 97.655 & \\
\hline 125 & \multicolumn{2}{|c|}{ Perf1_1... 1013.598} & 1004.688 & 97.663 & \\
\hline 126 & \multicolumn{2}{|c|}{ Perf1_1_.. 1013.646} & 1004.673 & 97.672 & \\
\hline 127 & \multicolumn{2}{|c|}{ Perf1_1... 1013.694} & 1004.659 & 97.680 & \\
\hline 128 & \multicolumn{2}{|c|}{ Perf1_1... 1013.741} & 1004.644 & 97.689 & \\
\hline 129 & \multicolumn{2}{|c|}{ Perf1_1... 1013.789} & 1004.629 & 97.698 & \\
\hline 130 & \multicolumn{2}{|c|}{ Perf1_1 ... 1013.837 } & 1004.615 & 97.706 & \\
\hline 131 & \multicolumn{2}{|c|}{ Perf1_1... 1013.885} & 1004.600 & 97.715 & \\
\hline 132 & \multicolumn{2}{|c|}{ Perf1_1.. 1013.933} & 1004.586 & 97.723 & \\
\hline 133 & \multicolumn{2}{|c|}{ Perf1_1... 1013.981} & 1004.571 & 97.732 & \\
\hline 134 & \multicolumn{2}{|c|}{ Perf1_1 ... 1014.028 } & 1004.556 & 97.740 & \\
\hline 135 & & 1004.542 & 97.749 & \\
\hline 136 & \multicolumn{2}{|c|}{ 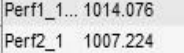 } & 1005.867 & 97.225 & \\
\hline 137 & \multicolumn{2}{|c|}{ Perf2_2 1007.259} & 1005.832 & 97.234 & \\
\hline 138 & \multicolumn{2}{|c|}{ Perf2_3 1007.295} & 1005.796 & 97.234 & \\
\hline 139 & \multicolumn{2}{|c|}{ Perf2_4 1007.330} & 1005.761 & 97.234 & \\
\hline 140 & \multicolumn{2}{|c|}{ Perf2_5 1007.365} & 1005.725 & 97.234 & \\
\hline 141 & \multicolumn{2}{|c|}{ Perf2_6 1007.400} & 1005.690 & 97.242 & \\
\hline 142 & \multicolumn{2}{|c|}{ Perf2_7 1007.436} & 1005.655 & 97.242 & \\
\hline 143 & \multicolumn{2}{|c|}{ Perf2_8 $\quad 1007.471$} & 1005.619 & 97.242 & \\
\hline 144 & \multicolumn{2}{|c|}{ Perf2_9 1007.506} & 1005.584 & 97.251 & \\
\hline 145 & \multicolumn{2}{|c|}{ Perf2_10 1007.542} & 1005.548 & 97.251 & \\
\hline 146 & \multicolumn{2}{|c|}{ Perf2_11 1007.577} & 1005.513 & 97.251 & \\
\hline 147 & \multicolumn{2}{|c|}{ Perf2_12 1007.612} & 1005.477 & 97.259 & \\
\hline 1148 & \multicolumn{2}{|c|}{ Perf? $1.3 \quad 1007647$} & $\frac{1005.542}{I I I}$ & 97259 & $r$ \\
\hline
\end{tabular}

Figura 8: Coordenadas de todos os traços de todos os perfis do relevo submerso.

\subsection{Interpolação dos dados}

Para a interpolação e geração do modelo digital de elevação (MDE) foi utilizado o interpolador Topo to Raster disponível no software ArcGIS por apresentar melhor RMS para a caixa d'água de acordo com o estudo realizado por (Carmo, 2014). Como não foi possível amostrar com o GPR as bordas interna e externa da caixa d'água, por causa das dimensões do barco usado para transportar as antenas, foram utilizadas as coordenadas obtidas com a estação total para compor os dados a serem interpolados.

\subsection{Comparação empregando pontos medidos com a topografia}

Como não se têm coordenadas observadas com a estação total coincidentes com os traços do radargrama, e sabendo que a caixa d'água investigada possui uma simetria geométrica muito regular, como é possível observar pelos dados medidos com a estação total e Figuras 3a e 9, foi adotado o

Bol. Ciênc. Geod., sec. Artigos, Curitiba, v. 23, nำ1, p.39 - 54, jan - mar, 2017. 
critério de realizar a comparação da profundidade a partir da média dos pontos observados pela estação total ajustados em circunferências, a partir da borda central da caixa.

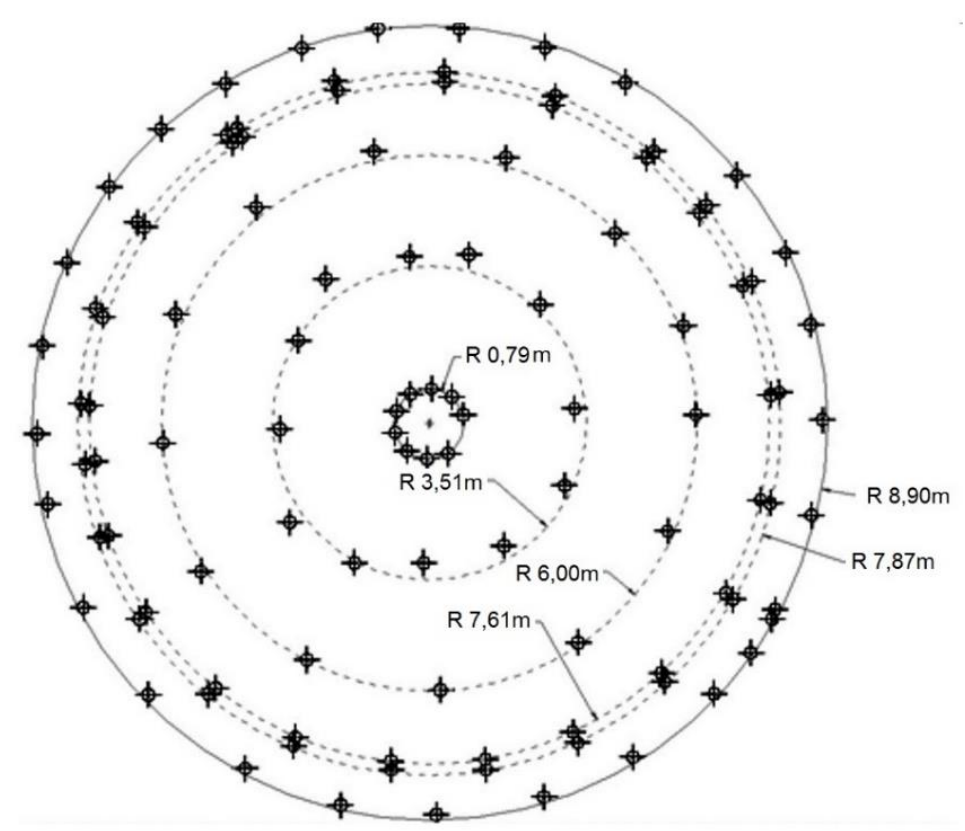

Figura 9: Disposição dos pontos observados na caixa d’água (ETA) com a estação total, com diferentes raios de circunferência ajustados aos pontos.

A Tabela 1 apresenta uma análise exploratória das profundidades levantadas com estação total próximas às circunferências, com o objetivo de verificar a regularidade geométrica observada em campo.

Tabela 1: Análise exploratória das profundidades levantadas com estação total.

\begin{tabular}{l|l|l|l|l}
\hline & \multicolumn{4}{|l}{ Profundidade na circunferência a partir da Borda } \\
\hline Raios a partir do centro da ETA & $\mathbf{R ~ 3 , 5 1 ~ m}$ & $\mathbf{R ~ 6 , 0 0 ~} \mathbf{~ m}$ & $\mathbf{R ~ 7 , 6 1 ~ m}$ & $\mathbf{R 7 , 8 7 ~ m}$ \\
\hline Menor profundidade observada (m) & 3,50 & 3,36 & 3,22 & 3,07 \\
\hline Maior profundidade observada (m) & 3,52 & 3,37 & 3,24 & 3,08 \\
\hline Amplitude (m) & 0,02 & 0,01 & 0,02 & 0,01 \\
\hline Média (m) & 3,51 & 3,36 & 3,23 & 3,08 \\
\hline Desvio padrão (m) & 0,005 & 0,003 & 0,005 & 0,004 \\
\hline
\end{tabular}

Pode-se observar que o maior desvio padrão foi de $5 \mathrm{~mm}$ e uma amplitude máxima igual a $2 \mathrm{~cm}$, mostrando que a média representa bem os valores observados para as circunferências. Portanto, a análise será realizada a partir das discrepâncias entre as profundidades médias contidas na Tabela $1 \mathrm{e}$ as profundidades extraídas dos radargramas nessas mesmas circunferências.

\subsection{Comparação entre MDE's}

De acordo com Li (1988), uma das maneiras de comparação entre dois modelos de elevação é considerar como variável randômica a diferença de altura $(\Delta h)$ entre os modelos digitais de elevação. Para isso ele sugere a coleta de amostras sobre os MDE's para a estimativa de toda a superfície. Para a obtenção das variáveis randômicas foi criada uma malha regular, com espaçamento de 1 metro, totalizando 241 pontos e computadas as discrepâncias $\Delta h_{i}$ entre as cotas empregando a Equação 3. 


$$
\Delta \mathrm{h}_{\mathrm{i}}=\mathrm{Z}_{\text {Ref }}\left(\mathrm{l}_{\mathrm{i}}\right)-\mathrm{Z}_{G P R}\left(\mathrm{l}_{\mathrm{i}}\right)
$$

Sendo:

$\mathrm{Z}_{\mathrm{GPR}}\left(\mathrm{l}_{\mathrm{i}}\right)$ : valor estimado para a profundidade na posição $l_{\tilde{i}}$, extraído do MDE (GPR);

$\mathrm{Z}_{\mathrm{Ref}}\left(\mathrm{l}_{\mathrm{i}}\right)$ : valor observado para a profundidade na posição $l_{\hat{i}}$, extraído do MDE (estação total).

Considerando $\Delta$ como uma variável randômica alguns parâmetros estatísticos podem ser calculados (Li, 1988):

Amplitude, diferença entre os extremos da discrepância:

$$
\mathrm{R}=\Delta h_{\text {máx }}-\Delta h_{\text {min }}
$$

Uma caraterística dessa medida é que ela leva em consideração apenas dois valores de $\Delta h$, descartando todos os outros.

Média das discrepâncias, medida de tendência central:

$$
\bar{d}=\frac{1}{n} \sum_{i=1}^{n} \Delta h_{i}
$$

Desvio padrão da amostra de discrepâncias, medida de dispersão:

$$
S d=\sqrt{\frac{1}{n} \sum_{i=1}^{n}\left(\Delta h_{i}-\bar{d}\right)^{2}}
$$

Um estimador que pode ser utilizado para descrever um conjunto de dados, independente da sua distribuição de probabilidade, é o RMS. Os outliers presentes no conjunto de dados devem ser eliminados uma vez que o valor do RMS é influenciado por eles. Este estimador retorna a incerteza presente no dado espacial avaliado, expondo a magnitude da discrepância que ocorre em campo (Santos, 2010).

\section{Raiz da média dos quadrados das discrepâncias $\left(\mathrm{RMS}_{\Delta \mathrm{h}}\right)$ :}

$$
R M S_{\Delta h}=\sqrt{\frac{1}{n} \sum_{i=1}^{n}\left(\Delta h_{i}\right)^{2}}
$$

Segundo Li (1988), uma combinação da média das discrepâncias com o desvio padrão da amostra pode ser usada como uma medida da acurácia, $A c_{y}$ do MDE.

$$
\mathrm{Ac}=\overline{\mathrm{d}} \pm \mathrm{Sd}
$$

De acordo com Li (1988), esta medida possui as seguintes características: a média representa uma translação da superfície gerada em relação à referência. Ela pode ser devido à inexatidão nas coincidências das posições $l_{i}$ para $Z_{\text {Ref e }} Z_{\mathrm{GPR}}$, Equação (3), bem como à efeitos sistemáticos. Se for nula, Ac é igual ao RMS. O desvio padrão Sd, mostra o quão bem o MDE se ajustou às observações de referência. 


\subsection{Cálculo do volume}

O MDE gerado por ferramentas do ArcGIS foi importado pelo "GPR Bathymetry" no módulo para cálculo de volume. Foram inseridos os limites interno e externo da caixa d'água para o cálculo correto do volume. As Figuras 10 e 11 mostram a interface desenvolvida, os MDE's e os valores dos volumes obtidos respectivamente pelos métodos utilizando a estação total e GPR.

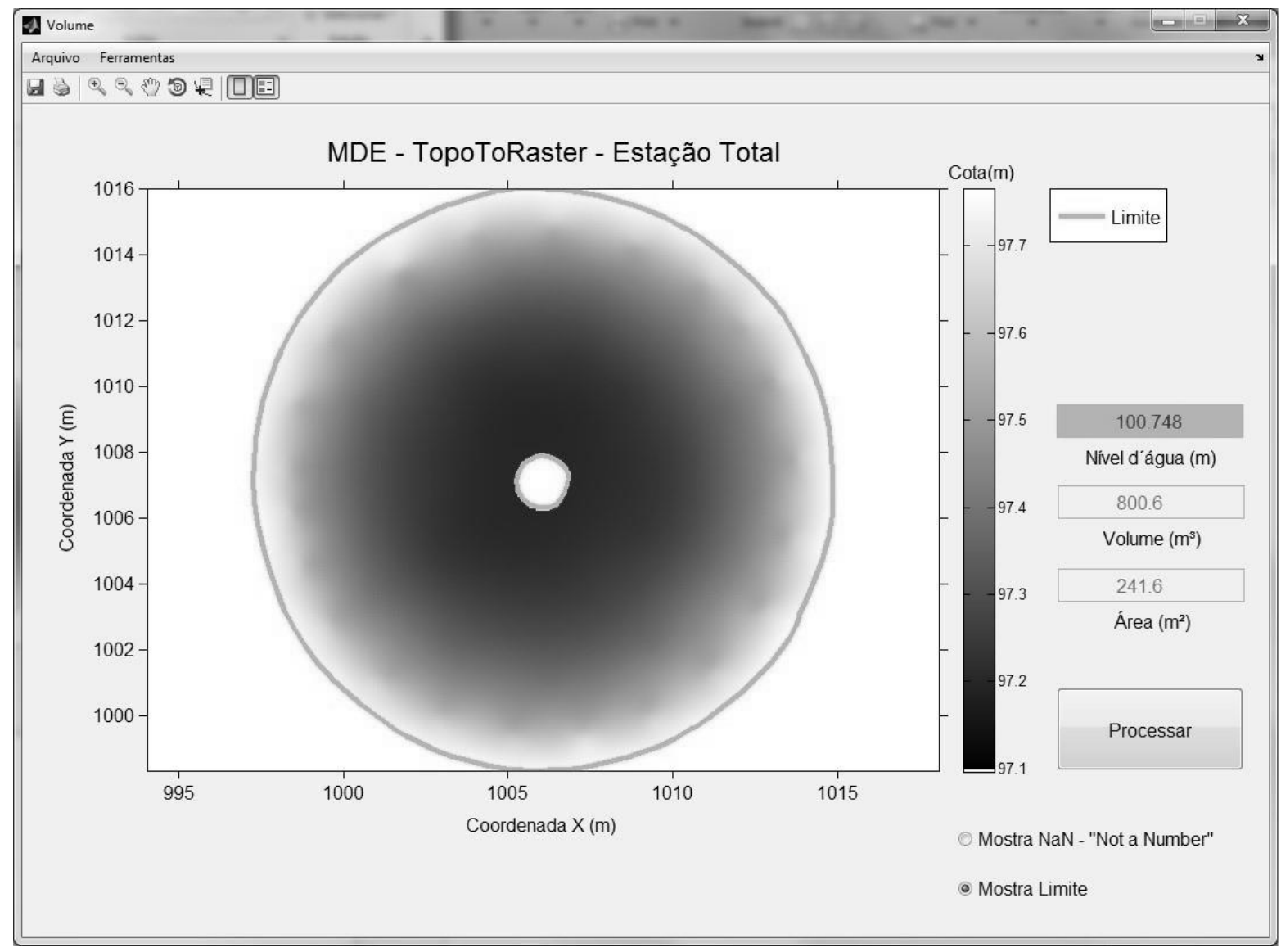

Figura 10: MDE gerado pelo interpolador Topo to Raster utilizando os pontos da Estação Total. 


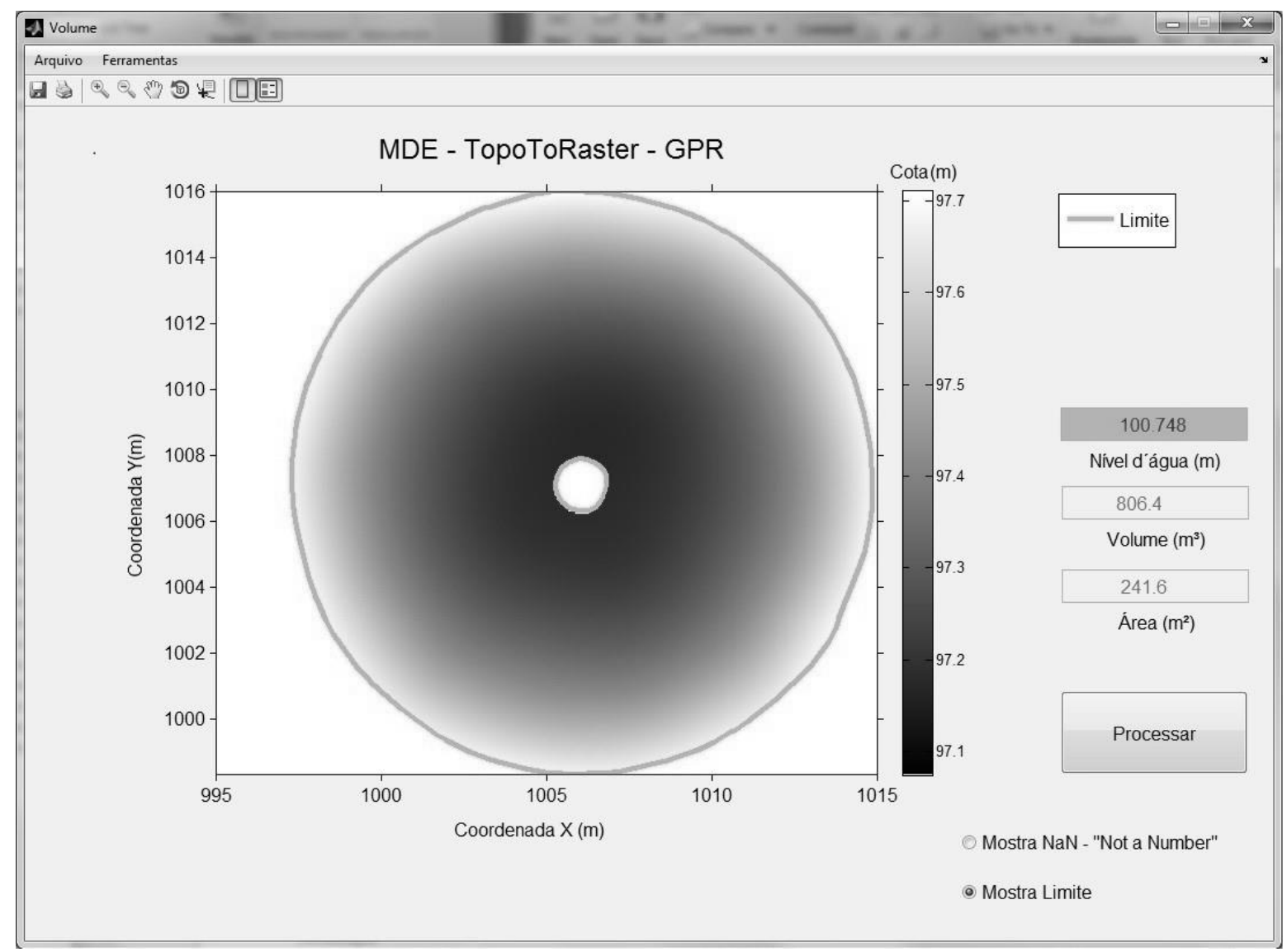

Figura 11: MDE gerado pelo interpolador Topo to Raster utilizando os pontos obtidos de modo manual (visual) com o GPR.

\section{Resultados e Discussões}

Para a primeira análise, tomou-se inicialmente os pontos observados pelo GPR na posição mais próxima das circunferências em estudo, gerando a Tabela 2. Enquanto com o levantamento topográfico as amplitudes foram menores que $2 \mathrm{~cm}$ e os desvios menores que $5 \mathrm{~mm}$, com o levantamento por GPR as amplitudes chegaram a $10 \mathrm{~cm}$ e os desvios $3 \mathrm{~cm}$.

Tabela 2: Analise exploratória das radiais levantadas com GPR.

\begin{tabular}{l|l|l|l|l}
\hline & \multicolumn{4}{|l}{ Profundidade a partir da Borda interna (metros) } \\
\hline Raios a partir do centro da ETA & $\mathbf{R ~ 3 , 5 1 ~ m}$ & $\mathbf{R ~ 6 , 0 0 ~} \mathbf{~ m}$ & $\mathbf{R ~ 7 , 6 1 ~} \mathbf{~ m}$ & $\mathbf{R 7 , 8 7 ~ m}$ \\
\hline Menor profundidade observada (m) & 3,54 & 3,37 & 3,25 & 3,24 \\
\hline Maior profundidade observada (m) & 3,47 & 3,35 & 3,22 & 3,14 \\
\hline Amplitude (m) & 0,07 & 0,02 & 0,03 & 0,10 \\
\hline Média (m) & 3,49 & 3,36 & 3,24 & 3,16 \\
\hline Desvio padrão (m) & 0,02 & 0,01 & 0,01 & 0,03 \\
\hline
\end{tabular}

Para comparar as médias obtidas pelos pontos amostrados com a estação total e as obtidas pelo georadar, para as circunferências de estudo, elaborou-se a Tabela 3. Onde pode-se verificar os valores para as discrepâncias das médias. A maior discrepância foi de $-8 \mathrm{~cm}$ enquanto a menor foi de $0 \mathrm{~cm}$. 
Tabela 3: Comparação das profundidades obtidas com o GPR com e com a estação total.

\begin{tabular}{l|l|l|l|l}
\hline & \multicolumn{4}{|c}{ Profundidade a partir da Borda interna (metros) } \\
\hline Raios a partir do centro da ETA & $\mathbf{R ~ 3 , 5 1 ~ m}$ & $\mathbf{R ~ 6 , 0 0 ~} \mathbf{~ m}$ & $\mathbf{R ~ 7 , 6 1 ~} \mathbf{~ m}$ & $\mathbf{R 7 , 8 7} \mathbf{~ m}$ \\
\hline Média (Perfis GPR) $(\mathrm{m})$ & 3,49 & 3,36 & 3,24 & 3,16 \\
\hline Média (Estação Total) $(\mathrm{m})$ & 3,51 & 3,36 & 3,23 & 3,08 \\
\hline Discrepância das médias $(\mathrm{m})$ & 0,02 & 0,00 & $-0,01$ & $-0,08$ \\
\hline
\end{tabular}

Para a segunda análise, onde se comparou o MDE gerado pela topografia com o MDE gerado pelo GPR, foi aplicado inicialmente o boxplot para a remoção de 4 outliers, identificados num universo de 241 pontos gerados por uma malha regular espaçada de $1 \mathrm{~m}$ em $1 \mathrm{~m}$. Em seguida foi realizada a análise estatística dos dados com base nas discrepâncias entre os MDE's (Tabela 4).

Tabela 4: Análise estatística da amostra de discrepâncias.

\begin{tabular}{l|c}
\hline \multicolumn{1}{c|}{ Índices estatísticos } & MDE (Estação Total) x MDE (GPR) \\
\hline$\Delta h_{\operatorname{máx}}(m)$ & 0,01 \\
\hline$\Delta h_{\min }(m)$ & $-0,08$ \\
\hline Amplitude $(\mathrm{m})$ & 0,09 \\
\hline Média (m) & $-0,02$ \\
\hline Desvio padrão (m) & 0,02 \\
\hline RMS (m) & 0,03 \\
\hline
\end{tabular}

Na Tabela 4 se verifica que a amplitude das discrepâncias entre os MDE's foi de $9 \mathrm{~cm}$, a média de -2 $\mathrm{cm}$, desvio da amostra de $2 \mathrm{~cm}$ e o RMS das discrepâncias de $3 \mathrm{~cm}$, levando a uma acurácia estimada de $4 \mathrm{~cm}$ pela Equação 8 .

Na comparação entre os MDE's o mapa de discrepâncias apresentado pela Figura 12, mostra que os pontos situados antes do início da elevação abrupta da caixa (Raio 7,61 m) não são muito discrepantes entre si e que as maiores discrepâncias ocorrem nas bordas.

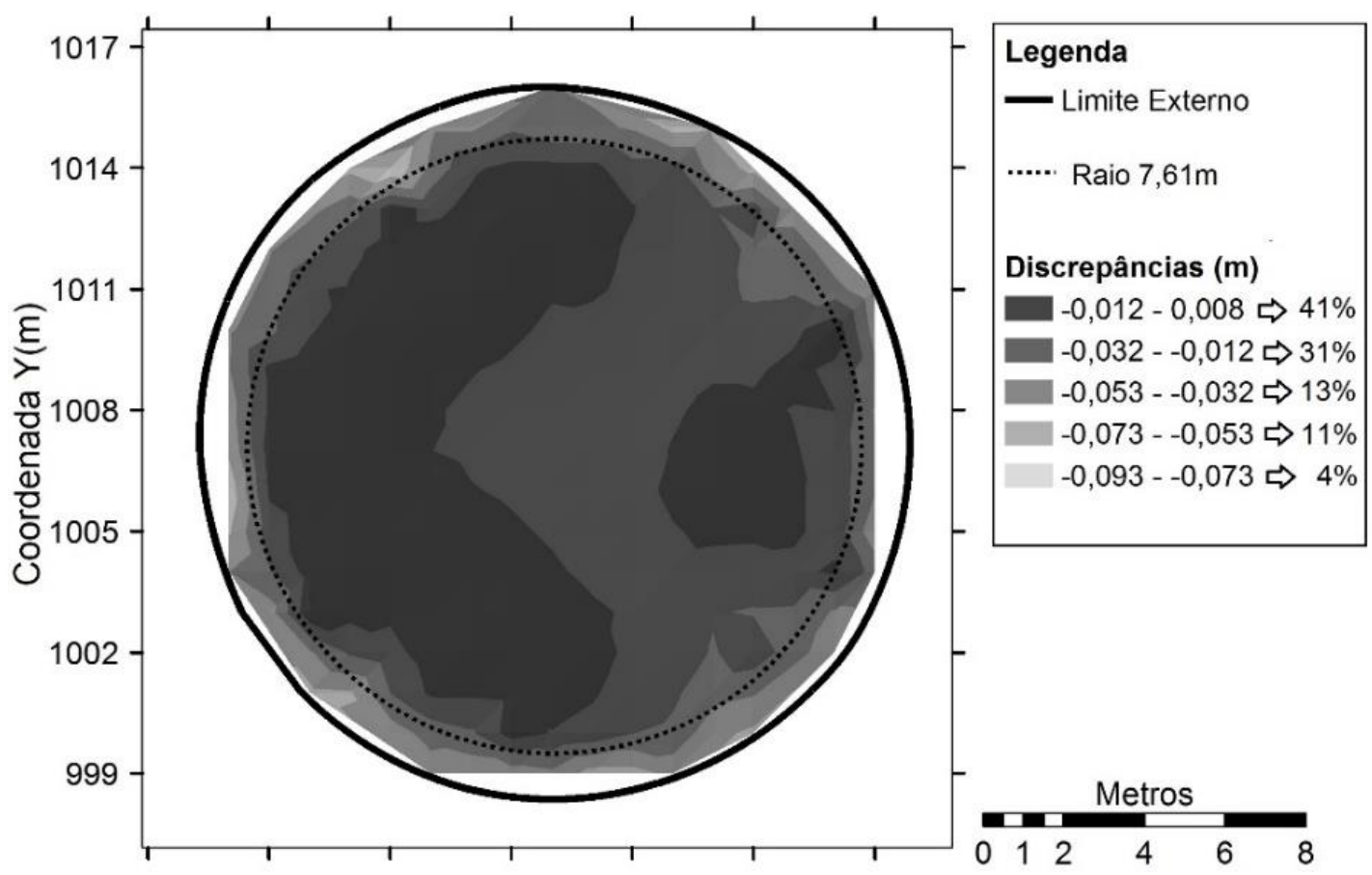

Figura 12: Mapa das discrepâncias. 
Ao observar o mapa de discrepâncias pode-se dizer que $72 \%$ dos dados possuem discrepâncias entre $1 \mathrm{~cm}$ a $-3 \mathrm{~cm}$, mostrando ser este um modelo consistente para essa faixa. Acredita-se que o aumento da discrepância nas bordas pode ser em função da distribuição espacial dos pontos e de efeitos de borda (edge effects) oriundos da interpolação do gride. Pode-se observar nas Figuras 4 e 9 que os pontos obtidos com o GPR não possuem uma distribuição espacial regular, estando mais próximos junto ao centro da ETA e mais afastados à medida que se aproximam da borda externa. Já os pontos levantados pela estação total estão mais bem distribuídos espacialmente.

Comparando os volumes determinados com os MDE's, observa-se que os volumes encontrados pelos dois métodos foram de $800,6 \mathrm{~m}^{3}$ para a estação total e $806,4 \mathrm{~m}^{3}$ para o GPR, o que corresponde a uma diferença de $1 \%$.

\section{Conclusões}

A comparação dos radargramas com as circunferências ajustadas aos pontos obtidos pela estação total, tomando-se a variável $\mathrm{Z}$ como a média aritmética desses pontos, apresentou resultados satisfatórios. Três, das quatro referências de comparação, apresentaram valores de discrepâncias inferiores a $2 \mathrm{~cm}$ e duas delas apresentou o valor $0 \mathrm{~cm}$.

Quando analisados os dois modelos digitais de elevação observa-se uma discrepância inferior a 0,03 m para $72 \%$ da área mapeada e um RMS para toda a superfície também de 0,03 m. Atribui-se o aumento da discrepância, à medida que se afasta da borda central da ETA, ao aumento do espaçamento entre os perfis que foram obtidos de maneira radial. Neste sentido, um ponto limitante na obtenção dos dados de GPR foi a distribuição não uniforme (Figura 4).

Os resultados obtidos no cálculo de volume se mostraram muito promissores, uma vez que se obteve uma diferença de $1 \%$ quando comparado com uma técnica tradicional e de melhor acurácia.

O objetivo de construir um aplicativo para acesso gratuito, visando a manipulação de dados de GPR foi alcançado com sucesso. Também foi possível realizar alguns processamentos básicos como: remove background; set time zero; edição do radargrama com linhas e polígonos; espacialização do radargrama em x, y e z; cálculo de velocidade de propagação; conversão do eixo y de tempo para distância; cálculo de áreas e volumes, dentre outros. Já estão sendo implementadas outras funcionalidades, como interpoladores para geração de MDE's e classificadores semiautomáticos.

O GPR se mostrou uma ferramenta multidisciplinar, isto é, uma ferramenta que apesar de ter sido inicialmente desenvolvida para trabalhos geológicos, apresentou-se como uma boa alternativa para trabalhos em tanques e reservatórios rasos confinados, como pode ser verificado para os resultados dos experimentos neste trabalho.

Para um reservatório de uma estação de tratamento de água construído em concreto armado, com profundidades variando de 3 a 3,5 metros, o levantamento do relevo submerso empregando um georadar, GPR, resultou em um modelo digital de elevação com acurácia estimada de $4 \mathrm{~cm}$, com as discrepâncias podendo chegar a $10 \mathrm{~cm}$. 


\section{AGRADECIMENTOS}

$\mathrm{O}$ autor externa seus agradecimentos à UFMG e ao professor Paulo Roberto Antunes Aranha por ceder o GPR e ajudar na coleta de dados, bem como a Edilson José do Carmo pela colaboração e disponibilização dos dados referentes ao levantamento topográfico da ETA.

\section{REFERÊNCIAS BIBLIOGRÁFICAS}

Annan, A. P. "Ground penetrating radar: Principles, procedures \& applications". Sensors \& Software Inc. Technical Paper. 2003. Acesso em 10 de julho, 2015. http://wwwrohan.sdsu.edu/ geology/jiracek/sage/documents/Sensors\%20and\%20Software\%20GPR\%20Manual.p df.

Aranha, P. R. A., Augustin, C. H. R. R. and Sobreira, F. G. "The use of GPR for characterizing underground weathered profiles in the sub-humid tropics." Jornal of Applied Geophysics, 2002.

Blondel, P. and Murton, B.J. Handbook of Seafloor Sonar Imagery. Eastergate, Chicester, West Sussex, England: Praxis Publishing Ltd . Hardcover, 75 Sterling. ISBN 0-471- 96217-1. 1997.

Botelho, M. A. B. "Modelamento Sísmico na Bacia do Recôncavo usando a Técnica de Traçamento de Raios". Tese de Doutorado. Programa de Pesquisa e Pós-Graduação em Geofísica, UFBA. 1986.

Carmo, E. J. "Avaliação dos interpoladores krigagem e topo to raster na geração de modelos digitais de elevação a partir de dados batimétricos". Dissertação de Mestrado. Universidade Federal de Viçosa. Viçosa, Minas Gerais, 2014.

Castelo Branco, R. M. G., and Castro, D. L. "Ground penetrating radar monitoring of a hydrocarbon leakage site in Fortaleza (Brazil) during its remediation process.” Journal of Applied Geophysics. 2003.

Faria, S. H. "O uso do georadar na determinação de parâmetros da estrutura de pavimentos flexíveis". Dissertação de mestrado. UFV - Universidade Federal de Viçosa. Viçosa - MG. 125p. 2010.

Gandolfo, O. C. B. "Um estudo do imageamento geoelétrico na investigação rasa. Tese de Doutorado. Programa de Pesquisa e Pós-Graduação em Recursos Minerais e Hidrogeologia, USP. 2007.

Li, Zhilin. "On the measure of digital terrain model accuracy." University of Glasgow. Photogrammetric Record, 1988: pags: 873-877.

Saarenketo, T. "Electrical properties of road materials and subgrade soils and the use of ground penetrating radar in traffic infrastructure surveys". PhD thesis: Faculty of Science, Department of Geoscience, University of Oulu, P.O. Box 3000, FI-90014. Edição: Professor Olli Vuolteenaho. Oulu: Oulu University Prees, 2006.

Santos, A. P. "Avaliação da Acurácia Posicional em Dados Espaciais com o uso de Estatística Espacial." Dissertação de mestrado. Universidade Federal de Viçosa. Viçosa, Minas Gerais, Brasil, 2010.

Shiraiwa, S., Bertol, G. A., and Hirooka, E. S. S. "Aplicação de GPR na localização de artefatos arqueológicos na Casa Dom Aquino em Cuiabá, MT." In: $1^{\circ}$ Simpósio de Geofísica, São Paulo. Anais do I Simpósio de Geofísica. São Paulo: Regional Sul da SBGF. v. CD-ROM. p. 1-4., 2004.

Recebido em 05 de novembro de 2015.

Aceito em 21 de março de 2016 